\title{
Les récepteurs couplés aux protéines G
}

\section{Des régulateurs allostériques du métabolisme cellulaire}

$>$ II y a environ cinquante ans, l'isolement des premières enzymes et l'analyse de leur mécanisme réactionnel ont mis les biochimistes au défi de comprendre le fonctionnement des enzymes régulatrices. Différents modèles fondés sur des informations pharmacologiques, enzymatiques et structurales ont été proposés. Parmi ceux-ci, le modèle dit allostérique de Monod, Wyman et Changeux décrit des protéines régulatrices qui peuvent adopter plusieurs conformations, interconvertibles et différemment stabilisées par les substrats, les produits et les effecteurs allostériques. Ces derniers interagissent au niveau de sites régulateurs topographiquement distincts du site enzymatique. Les divers états conformationnels associent des propriétés fonctionnelles et structurales distinctes. Enfin, la nature oligomérique des protéines qui ont servi à construire ce modèle a permis de décrire un phénomène important, la coopérativité. Celle-ci reflète la capacité d'une molécule liée à une sous-unité de la protéine à faciliter ou à défavoriser la liaison d'une deuxième molécule sur une sous-unité voisine. Ce concept d'allostérie a évolué. II est à présent étendu aux phénomènes de modulation allostérique qui mettent en jeu des molécules distinctes, se liant à leurs sites propres sur une protéine monomérique, comme par exemple un récepteur couplé aux protéines $G$. Cet article a pour objectif de discuter la manière dont les récepteurs couplés aux protéines $G$, d'une part, s'inscrivent dans un modèle d'architecture fonctionnelle répondant aux règles de l'allostérie et, d'autre part, sont soumis à une modulation de leurs propriétés pharmacologiques et fonctionnelles par des effecteurs allostériques, des petites molécules ou des protéines cellulaires, avec lesquels ils établissent des contacts stables ou transitoires. <

\section{À la croisée des chemins entre RCPG et allostérie}

Les récepteurs couplés aux protéines $\mathrm{G}(\mathrm{RCPG})$ forment une superfamille de protéines membranaires monomériques de près de 900 membres [1]. Globalement impliqués dans les phénomènes de communication et d'homéostasie cellulaire, ils interviennent dans de nombreuses fonctions physiologiques et physiopathologiques et représentent des cibles thérapeutiques importantes. Des efforts de recherche soutenus ont permis d'enrichir un arsenal thérapeutique considérable composé d'agonistes (stabilisant un état actif) et d'agonistes inverses (stabilisant un état inactif) de ces récepteurs $[2,31,32](\rightarrow)$.

Notons que la majorité des molécules décrites et utilisées en tant qu'antagonistes sont pro-

$(\rightarrow)$ Voir les synthèses de J. Sebag et J. Pantel (page 845), et G. Lebon et C.G. Tate, page 876 de ce numéro bablement des agonistes inverses. La plupart de ces ligands ont été sélectionnés sur la base d'une interaction compétitive avec la liaison de l'agoniste endogène au niveau de son site récepteur (site orthostérique). Ces molécules orthostériques ont souvent des affinités très élevées mais ne sont pas dénuées d'effets secondaires liés en partie à leur manque de sélectivité. En effet, de nombreux récepteurs coexistent sous forme de sous-types distincts qui présentent de fortes homologies de séquence au niveau de leur site orthostérique [1]. D'autre part, il est à présent bien reconnu qu'un même agoniste (et a fortiori des agonistes de structures chimiques différentes) peut sélectionner des voies de signalisation intracellulaires variées dépendantes du contexte cellulaire ou de la réponse biologique considérée [3]. 
A

Modèle d'activation

à deux états conformationnels

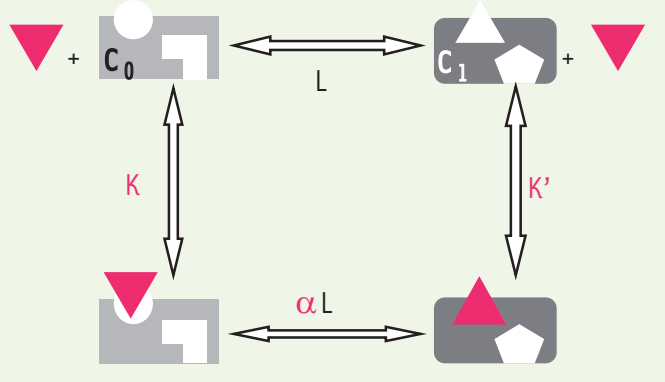

B

Modèle de modulation allostérique

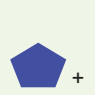

$c_{0}$ $c_{0}$

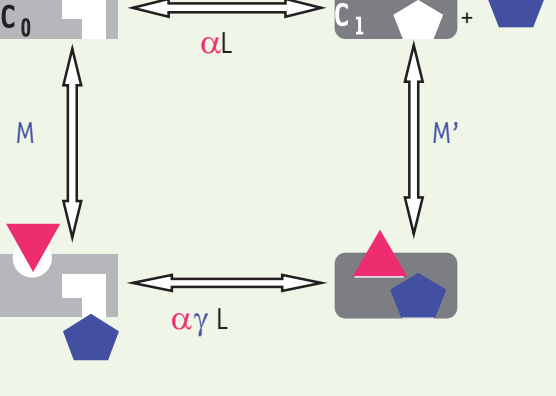

Figure 1. Modèles d'activation et de modulation allostérique d'un récepteur.

A. Modèle d'activation à deux états conformationnels. Le récepteur peut adopter deux conformations, $C_{0}$ (gris clair ; inactif) ou $C_{1}$ (gris foncé ; actif). L'équilibre spontané entre ces états conformationnels est régi par

la constante d'isomérisation $\mathrm{L}$ égale à $\left[\mathrm{C}_{0}\right] /\left[\mathrm{C}_{1}\right]$. Un agoniste (triangle rouge) distingue les deux états sur le plan de l'affinité (constantes de dissociation à l'équilibre $K$ ou $K^{\prime}$ ). Il occupe préférentiellement la conformation active pour laquelle il présente la meilleure affinité et déplace l'équilibre entre $C_{0}$ et $C_{1}$. L'efficacité intrinsèque $\alpha$ du ligand (rapport d'affinité $\alpha=K^{\prime} / K$ ) se conjugue à la constante d'isomérisation pour définir le nouvel équilibre entre $C_{1}$ et $C_{0}(\alpha \times L)$. Un agoniste $(K$ ' $K ; \alpha<1 ; \alpha L<L)$ déplace l'équilibre en faveur de l'état $C_{1}$. Un antagoniste ( $\left.K=K^{\prime}\right)$ n'affecte pas l'équilibre conformationnel initial; un agoniste inverse ( $K$ ' $>K ; \alpha>1 ; \alpha L>L$ ) privilégie l'état inactif $C_{0}$. $B$. Modèle de modulation allostérique. Conformément à la description précédente, un effecteur allostérique (bleu) peut se lier de manière préférentielle (constantes de dissociation à l'équilibre $M$ ou M') à l'une des deux conformations présentées. Pour M' < $M$, il s'agira d'un effecteur allostérique positif (PAM), pour M' > M, il s'agira d'un effecteur allostérique négatif (NAM). L'effecteur allostérique conjugue son efficacité intrinsèque $\gamma$ (rapport M'/M) à celle de $L$ pour modifier l'équilibre entre $C_{0}$ et $C_{1}$ selon le produit $L \times \gamma$. La présence d'un agoniste (triangle rouge) modifie encore la valeur de l'équilibre entre $C_{0}$ et $C_{1}$ selon le produit $L \times \alpha \times \gamma$. Cette figure illustre le cas d'un modulateur positif $(\gamma<1)$ qui renforce l'activation du récepteur par l'agoniste.

Ainsi ont émergé : (1) l'idée de rechercher des composés ciblant des domaines de RCPG moins conservés, (2) la nécessité de comprendre les bases moléculaires sous-jacentes à la sélection d'états conformationnels et fonctionnels spécifiques pour chaque RCPG, et (3) la possibilité d'utiliser ces outils et ces connaissances pour atteindre un niveau supérieur de sélectivité et de régulation de l'activité physiologique de ces récepteurs. D'où l'intérêt croissant porté à la modulation allostérique des $\operatorname{RCPG}[3,4]$ et au modèle allostérique initialement décrit par Monod, Wyman et Changeux [5].

\section{États conformationnels multiples et voies de signalisation des RCPG}

Les phénomènes biologiques observés lors de l'activation des RCPG sont complexes. Leur interprétation est limitée par nos connaissances mécanistiques et structurales insuffisantes et par la difficulté d'établir un modèle opérationnel simple rendant compte des observations expérimentales. Le modèle le plus courant [6], représenté sous forme simplifiée dans la Figure 1, intègre la capacité du récepteur à osciller entre deux états (actif et inactif) et à lier simultanément plusieurs ligands différents (agoniste endogène, composés orthostériques ou allostériques, protéines régulatrices). Conformément aux requis du modèle MWC (Monod-Wyman-Changeux) [5], les deux états conformationnels sont dotés de propriétés de liaison et de fonctions distinctes. Leur équilibre spontané régit l'activité basale encore appelée activité constitutive du récepteur [7]. La liaison préférentielle d'un ligand à une conformation donnée s'établit selon des critères d'affinité et sous contrôle cinétique, et déplace l'équilibre en faveur de cet état. La formation d'un complexe ternaire (liaison de deux ligands à des sites topographiquement distincts) conduit à un nouvel équilibre et à des modifications réciproques des propriétés de liaison et de fonction de chacun des ligands. Ce modèle rend compte de diverses propriétés des molécules allostériques comme (1) leur capacité intrinsèque à générer (agoniste allostérique) ou non (modulateur allostérique) une réponse biologique, (2) la saturabilité de leur effet modulateur, (3) leur aptitude à modifier les paramètres d'affinité et d'efficacité d'un ligand orthostérique $[3,4,6]$. II n'en est pas moins vrai qu'un modèle à deux états ne peut pas rendre compte de la complexité des voies de signalisation des RCPG. De nombreuses études fonctionnelles suggèrent en effet l'existence d'états actifs multiples, stabilisés de manière différentielle par les ligands orthostériques (ou allostériques) et discriminés par des partenaires de signalisation intracellulaires différents $[3,8,9]$. Les données structurales obtenues à partir des RCPG cristallisés dans des états actif et inactif $[10,32](\rightarrow)$ complétées par des études biophysiques

$(\rightarrow)$ Voir la synthèse de G. Lebon et C.G. Tate, page 876 de ce numéro 


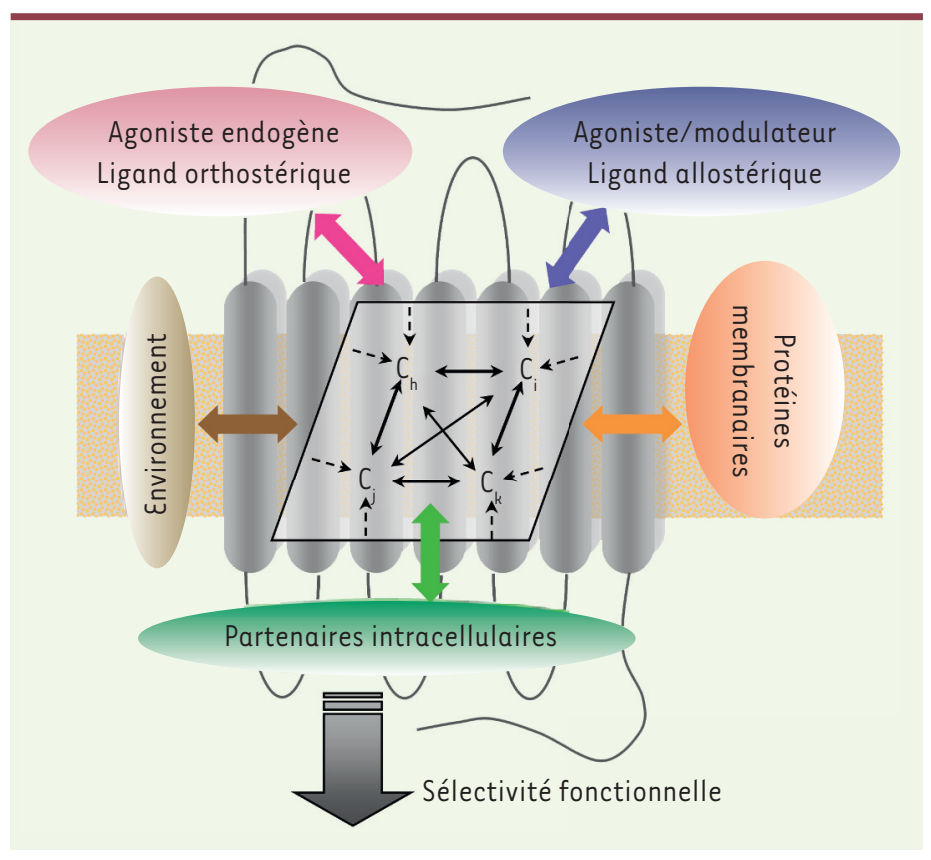

Figure 2. Modulation allostérique multifactorielle des RCPG. Au sein de son environnement membranaire naturel, le récepteur oscille entre de multiples états conformationnels $\left(C_{n}\right)$ interconvertibles. L'équilibre entre ces états actifs et inactifs est perturbé de manière différentielle et dynamique par de nombreux modulateurs allostériques ciblant des domaines topographiquement distincts du récepteur : la face extracellulaire (ligands orthostériques et allostériques, ions), l'interface protéine-membrane (lipides, protéines membranaires, récepteurs) ou la face intracellulaire (ions, partenaires de signalisation). L'intégration de l'ensemble de ces régulations allostériques permet la stabilisation d'états conformationnels actifs privilégiés et l'observation d'une sélectivité fonctionnelle.

récentes [11, 12], confortent une vue dynamique des RCPG, oscillant entre des états conformationnels multiples (Figure 2).

Un progrès notable dans l'analyse d'états actifs multiples a été accompli grâce à l'enregistrement synchronisé de la liaison d'agonistes à un récepteur et des réponses engendrées par cette liaison. L'utilisation d'une méthode de détection qui repose sur le principe du transfert d'énergie de fluorescence, un processus compatible avec les mesures de liaison en temps réel sur cellules vivantes, a été déterminante $[13,14,33](\rightarrow)$.

Elle a permis de montrer que le décapeptide neurokinine $A$, agoniste naturel du récepteur

$(\rightarrow)$ Voir la synthèse de J.L. Banères et B. Mouillac, page 837 de ce numéro NK2 des tachykinines, se lie rapidement à une conformation de faible affinité de ce RCPG, suivie par une transition lente vers un état conformationnel de plus haute affinité $[8,15,16]$. L'examen des réponses associées indique que la liaison rapide coïncide temporellement avec la libération de calcium intracellulaire (réponse de type Gq classiquement associée au récepteur NK2), tandis que la phase lente coïncide avec une réponse plus tardive, caractérisée par l'augmentation de la concentration intracellulaire d'AMP cyclique (AMPc, adénosine monophosphate cyclique). Fait intéressant, l'agoniste neurokinine A 4-10 (neurokinine A tronquée de ses trois premiers acides aminés) ne présente pas de phase de liaison lente et n'engendre pas non plus d'élévation d'AMPc $[8,16]$. Ces observations militent en faveur d'un modèle fonctionnel et structural (Figure $3 A$ ) décrivant un état inactif, un état actif précoce (responsable de la réponse calcique) et un état actif tardif (couplé à la production d'AMPc). Il permet d'expliquer comment des réponses distinctes (ou dissociables) peuvent résulter de la stabilisation (parfois transitoire) d'états conformationnels identifiés par leur affinité pour les ligands et par leur couplage à une voie de signalisation donnée. Ces travaux révèlent en outre que les messagers de la réponse cellulaire exercent un rétrocontrôle, positif ou négatif, sur le niveau d'activité du récepteur NK2. À titre d'exemple, l'activation de la voie $\mathrm{Gq}$ conduit à l'activation de la protéine kinase $C$, avec pour conséquence la réduction de l'amplitude de la réponse calcique et l'accélération de la transition vers l'état actif tardif. De même, la stimulation de l'activité de la protéine kinase A par I'AMPc accélère la transition vers l'état tardif. Ainsi, la modulation de l'effet d'un agoniste se liant sur la face extracellulaire d'un RCPG par des partenaires de signalisation intracellulaires reflète un mode de régulation typiquement allostérique [11, 16].

\section{Partenaires allostériques des RCPG}

Dans son acceptation littérale, un RCPG catalyse l'échange des nucléotides GDP/GTP au niveau de la sous-unité $\alpha$ des protéines $G$ hétérotrimériques, comme le font des protéines intracellulaires appelées guanine exchange factor (GEF) au niveau des petites protéines $G$ monomériques [17]. La stimulation d'un RCPG par un agoniste diminue l'affinité de G $\alpha$ pour le GDP et favorise son remplacement par du GTP. De manière réciproque, la protéine $\mathrm{G}$ (liée au GDP) stabilise un état de haute affinité pour les agonistes [18]. La modulation allostérique des RCPG s'étend bien au-delà des protéines $G$ puisque de très nombreux facteurs (ions, lipides, protéines, xénobiotiques), ciblant des domaines topographiquement distincts, modifient l'équilibre conformationnel des récepteurs et modulent leur couplage aux systèmes de signalisation intracellulaire (Figure 2).

Parmi les effecteurs allostériques naturels de RCPG, on peut citer brièvement les ions zinc, qui sont des modulateurs positifs du récepteur $\beta 2$-adrénergique [19], ou le cholestérol, qui module la fonctionnalité de nombreux récepteurs en se liant à des sites spécifiques non annulaires [20], et un ensemble de protéines appelées GIP (GPCR-interacting protein) [21], comprenant entre 


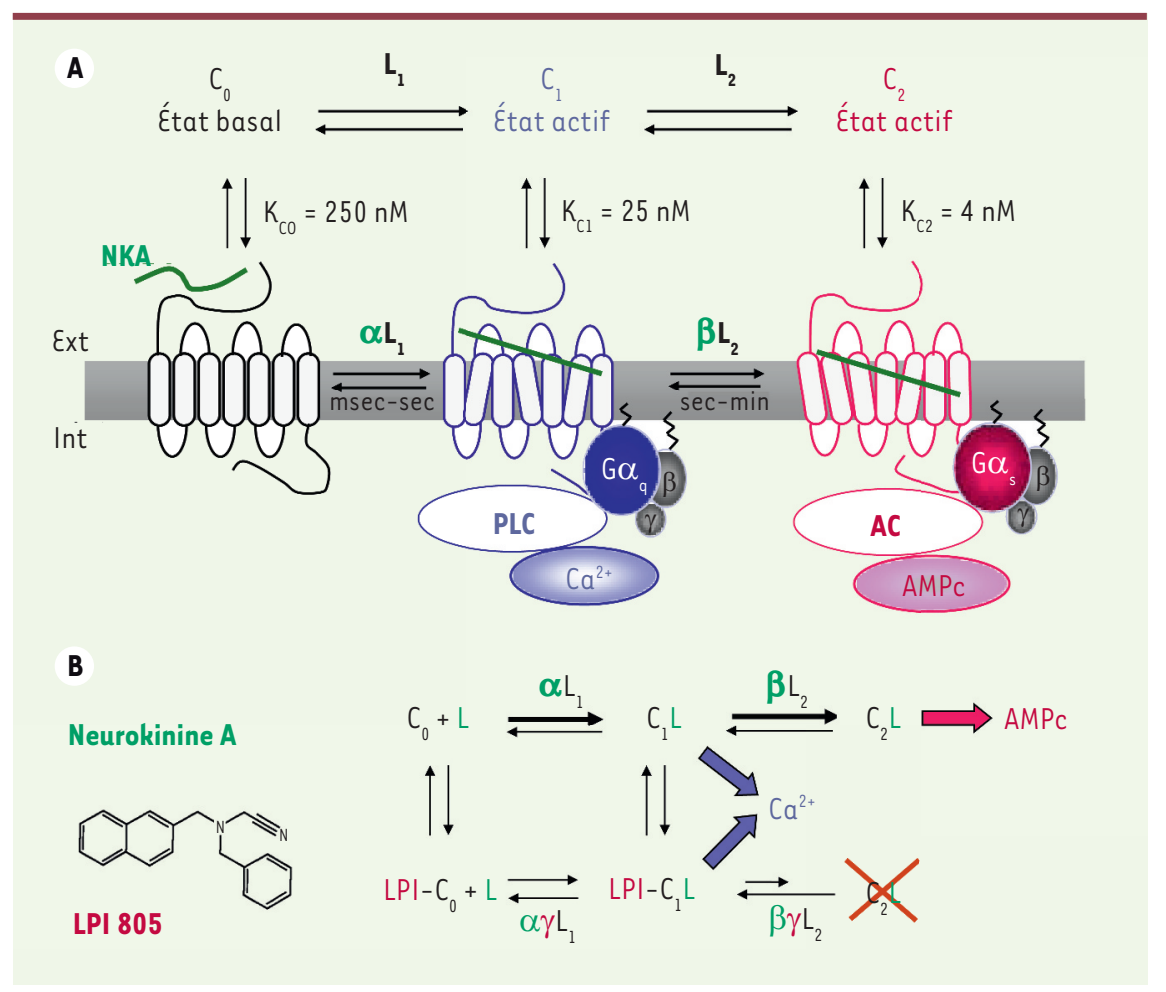

Figure 3. Dynamique conformationnelle et fonctionnelle du récepteur NK2 des tachykinines. $A$. Un modèle à trois états. Le récepteur NK2 oscille entre trois états conformationnels: $C_{0}, C_{1}$ et $C_{2}$. L'équilibre entre ces trois états interconvertibles est régi par les constantes d'isomérisation $\mathrm{L}_{1}$ (ou $\left[\mathrm{C}_{0}\right] /\left[\mathrm{C}_{1]}\right.$ ) et $L_{2}$ (ou $\left[C_{1}\right] /\left[C_{2}\right]$ ). La liaison de l'agoniste neurokinine $A$ (NKA) est caractérisée par une phase initiale rapide (processus bimoléculaire) conduisant à la stabilisation $\left(\alpha L_{1}\right)$ de l'état $C_{1}$ d'affinité intermédiaire. On observe ensuite une phase de liaison plus lente, qui correspond à l'isomérisation des complexes $C_{1} L$ en complexes $C_{2} L$ de plus haute affinité. La coïncidence temporelle entre les deux étapes de liaison et la production des signaux intracellulaires $\left(\mathrm{Ca}^{2+}\right.$ et $\left.\mathrm{AMPC}\right)$ a permis de discerner les états conformationnels et de les associer à des états fonctionnels $[8,16]$. AC : adénylate cyclase ; PLC : phospholipase C. B. Modulation allostérique de l'activation du récepteur NK2 des tachykinines. Le composé LPI 805 se lie au niveau d'un site distinct du récepteur et se comporte comme un inhibiteur partiel et non compétitif de la liaison de NKA $[15,27]$. Il stabilise la formation des complexes $C_{1} L$ au détriment de l'état $C_{2}$. Sur le plan fonctionnel, il bloque la réponse AMPc et potentialise la production de $\mathrm{Ca}^{2+}$. Le LPI 805 présente les propriétés d'un effecteur allostérique neutre ou positif sur la réponse calcique, et d'un effecteur allostérique négatif sur la production d'AMPc. Le résultat net est une dissociation des deux réponses associées à la NKA.

autres les arrestines, les protéines RAMP (receptor activity modifying proteins) et les pepducines, une nouvelle famille de peptides qui activent des RCPG par leur côté intracellulaire [22]. Les récepteurs eux-mêmes peuvent se comporter comme des modulateurs allostériques en formant des homo- ou des hétérodimères au sein desquels chaque monomère transfère de l'information vers l'autre pour conférer de nouvelles propriétés à l'ensemble [34] $\rightarrow$ ).

$(\rightarrow)$ Voir la synthèse de R. Jockers et al., page 864 de ce numéro

\section{L’exemple des récepteurs muscariniques et du récepteur NK2 des tachykinines}

En ce qui concerne les petites molécules de synthèse et les candidats médicaments [31] $(\rightarrow)$, les recherches foisonnent. Les premiers travaux

$(\rightarrow)$ Voir la synthèse de J.A. Sebag et J. Pantel, page 845 de ce numéro ont été menés sur les récepteurs muscariniques de l'acétylcholine qui appartiennent à la classe A des RCPG (Figure 4A) $[1,10]$. Ils localisent leur domaine de liaison orthostérique au sein des 7TM (7 domaines transmembranaires) et portent au moins deux sites allostériques distincts [4, 23]. De très nombreux modulateurs allostériques, qui incluent les molécules prototypes gallamine, brucine et W84, et d'autres molécules plus récentes, potentialisent ou inhibent la liaison et/ou la fonction des ligands muscariniques orthostériques $[4,23,24]$. Néanmoins, et à de rares exceptions près, tous ces ligands allostériques ralentissent (à des degrés divers) les cinétiques à la fois d'association et de dissociation des ligands orthostériques. À défaut de preuves directes de leur capacité à moduler l'équilibre conformationnel des récepteurs muscariniques, et compte tenu de la localisation du site allostérique à l'entrée de la gorge conduisant au site de l'acétylcholine (Figure 4A) [4, 35], il est fort probable que beaucoup de ces modulateurs agissent par encombrement stérique. D'autres molécules, tels I'AC-42, son analogue 77-LH-28-1 et le TBPB [24], tirent probablement leur remarquable sélectivité fonctionnelle d'une interaction avec un site allostérique peu conservé parmi les sous-types de récepteurs muscariniques. II n'est cependant pas exclu que certains de ces agonistes puissent chevaucher les domaines orthostérique et allostérique, selon un processus de liaison bitopique. Une telle possibilité, confortée par la proximité de ces domaines (Figure 4A) [35], fait actuellement l'objet de recherches intenses visant à l'obtention de molécules très affines et très sélectives [25, 26].

En ce qui concerne le récepteur NK2 des tachykinines, un autre RCPG de classe $A$, il a été montré que le modulateur allostérique LPI 805 affectait différemment les réponses engendrées par la neurokinine $A[15,27]$. En effet, il déstabilise l'état actif tardif couplé à la pro- 
A

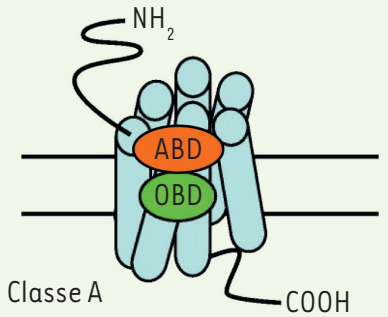

$\mathrm{COOH}$

Modulation allostérique ou barrière physique?

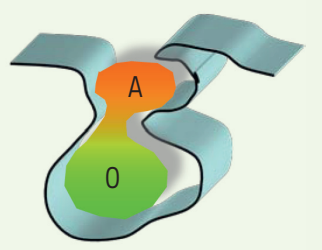

Liaison

bitopique
B

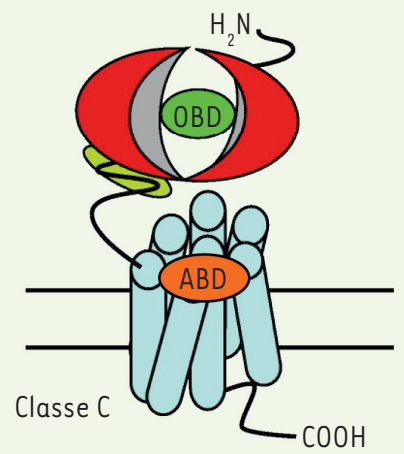

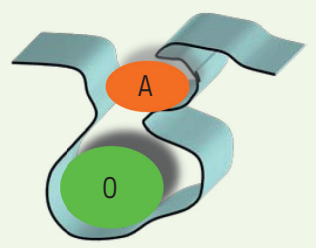

ou bariere physique?

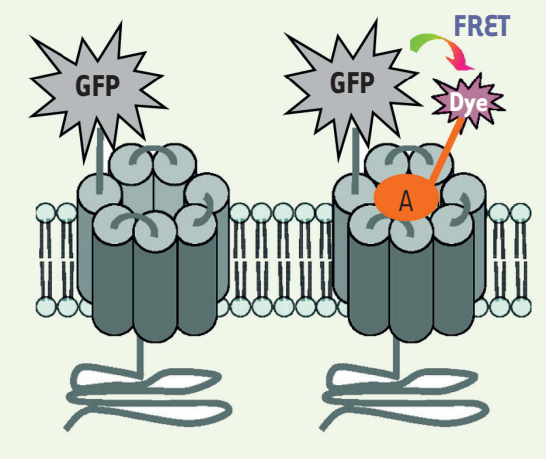

$\mathrm{OBD}$ : domaine de liaison de ligands orthostériques 0 $A B D$ : domaine de liaison de ligands allostériques $A$

Figure 4. Modulation allostérique des RCPG : questions et perspectives. $A$. Les RCPG de classe A qui ont un petit domaine amino-terminal, et en particulier les récepteurs de bioamines, se caractérisent par la proximité (voire un recouvrement partiel) de leurs domaines de liaison orthostérique $(O B D$, vert) et allostérique ( $A B D$, orange). Ceci complique considérablement la caractérisation de nouvelles molécules et la distinction entre modulateurs allostériques, inhibiteurs stériques ou molécules bitopiques [25, 26]. B. Les RCPG de classe C (comme les mGluR [metabotropic glutamate receptor]) présentent des domaines $O B D$ (module extracellulaire) et $A B D$ (domaines transmembranaires [TM]) plus éloignés. Après délétion de la partie amino-terminale, la partie transmembranaire de ces récepteurs montre une activité constitutive et une capacité d'activation directe par des ligands, comme le font les récepteurs de

classe A. En particulier, l'élimination du frein allostérique qu'est le domaine amino-terminal des mGluR confère aux modulateurs allostériques positifs une nature agoniste $[28,29]$. Ces observations sont à la base d'un essai en FRET (transfert d'énergie de fluorescence) permettant d'identifier, sur un récepteur mGluR tronqué et fusionné à la GFP (green fluorescent protein), de nouveaux ligands allostériques [30]. Leur validation en tant que modulateurs allostériques positifs se fait ensuite sur des récepteurs intacts, sur la base d'essais de liaison et d'essais fonctionnels.

duction d'AMPc et potentialise la libération de calcium intracellulaire, la réponse associée à l'état actif précoce (Figure 3B). II devient ainsi possible de disséquer les voies de signalisation associées à un récepteur afin de déterminer la contribution de chaque réponse dans des systèmes physiologiques intégrés.

\section{La quête d'effecteurs allostériques des RCPG}

De nombreux travaux fructueux ont permis l'identification d'effecteurs allostériques pour les RCPG de classe A (bioamines, adénosine, ghréline, chimiokines, neurokinines), de classe B (glucagon-like peptides, calcitonine gene-related peptide) et de classe C (GABA, calcium et glutamate) $[3,4,28]$. Les récepteurs de classe C (Figure 4B), contrairement aux RCPG de classe $A$, possèdent une longue extrémité amino-terminale, de structure caractéristique, qui abrite le site orthostérique et le maintient à distance des hélices transmembranaires où se logent les ligands allostériques [28]. L'expression de la seule partie transmembranaire de ces récepteurs produit une protéine fonctionnelle sur laquelle un effecteur allostérique positif du récepteur natif se comporte comme un agoniste [29]. Ces propriétés sont à présent mises à profit (Figure 4B) pour cribler des collections de molécules [30], en mode liaison et réponse, en vue de la sélection d'agonistes et de l'identification de nouveaux modulateurs positifs.

La quête d'effecteurs allostériques des RCPG se nourrit de réussites illustrées par l'usage thérapeutique : par exemple, l'usage thérapeutique du cinacalcet (modulateur positif de la liaison de calcium au récepteur CaSR [calcium-sensing receptor]) et du maraviroc (inhibiteur de la voie d'entrée du VIH impliquant le récepteur CCR5 des chimiokines) et l'existence de nombreuses molécules actuellement $\rightarrow \rightarrow$ Voir la synthèse de testées dans des essais cliniques J.A. Sebag et J. Pantel, [31] $(\rightarrow)$. page 845 de ce numéro

Elle se justifie par des objectifs majeurs que sont : (1) la compréhension et la maîtrise de la dynamique conformationnelle et fonctionnelle des RCPG, (2) la modulation fine et spatiotemporelle de l'activité d'un agoniste endogène in vivo, et (3) l'exploration de l'ensemble des surfaces et interfaces de ces récepteurs en vue de l'obtention de molécules permettant l'activation sélective de sous-types de récepteurs et le pilotage des voies de signalisation. 
L'exploitation des domaines allostériques des RCPG ouvre des champs d'investigation considérables, alliant recherche fondamentale et recherche clinique. $\diamond$

\section{SUMMARY}

\section{G protein-coupled receptors: allosteric regulators of cell} metabolism

Fifty years ago, the first successful isolation of enzymes and the study of their reaction mechanisms challenged biochemists to investigate their biological regulation. Various models have been proposed on the basis of available catalytical, pharmacological and structural information. The "allosteric model" of Monod, Wyman and Changeux describes regulatory proteins that can adopt multiple interconvertible conformations, differently stabilized by substrates, products and allosteric effectors. These effectors target regulatory sites topographically distinct from the enzymatic reaction center. Each conformational state is characterized by a unique set of pharmacological, functional and structural properties. The oligomeric nature of the proteins which were used to construct this model allowed to describe an important phenomenon, referred to as cooperativity. It explains how the binding of a molecule to one subunit of the protein can facilitate, or conversely impede, the binding of a second molecule to a neighboring subunit. This concept has evolved and now extends to allosteric regulatory phenomena dealing with distinct effectors that bind to their own sites on a monomeric protein, such as a G-protein coupled receptor. This article focuses on G-protein-coupled receptors and aims to discuss (1) how their functional architecture meets the rules of allostery, and (2) how allosteric effectors (small molecules or cell components), with which the receptors establish stable or transient interactions, may cooperate to finely tune their pharmacological and functional properties. $\diamond$

\section{LIENS D'INTÉRÊT}

Les auteurs déclarent n'avoir aucun lien d'intérêt concernant les données publiées dans cet article.

\section{RÉFÉRENCES}

1. Lagerström MC, Schiöth HB. Structural diversity of $G$ protein-coupled receptors and significance for drug discovery. Nat Rev Drug Discov $2008 ; 7$ : 339-57.

2. Imming $P$, Sinning $C$, Meyer A. Drugs, their targets and the nature and number of drug targets. Nat Rev Drug Discov $2006 ; 5: 821-34$.

3. Kenakin T, Miller LJ. Seven transmembrane receptors as shapeshifting proteins: The impact of allosteric modulation and functional selectivity on new drug discovery. Pharmacol Rev $2010 ; 62: 265-304$.

4. De Amici M, Dallanoce C, Holzgrabe U, et al. Allosteric ligands for G protein-coupled receptors: A novel strategy with attractive therapeutic opportunities. Med Res Rev $2010 ; 30: 463-549$.

5. Monod JJ, Wyman J, Changeux JP. On the nature of allosteric transition: a plausible model. J Mol Biol $1965 ; 12: 88-118$.

6. Hall DA. Modeling the functional effects of allosteric modulators at pharmacological receptors: Extension of the two-state model of receptor activation. Mol Pharmacol $2000 ; 58: 1412-23$.

7. Costa T, Cotecchia S. Historical review: Negative efficacy and the constitutive activity of G-protein-coupled receptors. Trends Pharmacol Sci $2005 ; 26: 618-24$.

8. Lecat S, Bucher B, Mely Y, Galzi JL. Mutations in the extracellular amino-terminal domain of the NK2 neurokinin receptor abolish cAMP signaling but preserve intracellular calcium responses. J Biol Chem $2002 ; 277$ : 42034-48.

9. Stallaert W, Dorn JF, van der Westhuizen $\varepsilon$, et al. Impedance responses reveal beta2-adrenergic receptor signaling pluridimensionality and allow classification of ligands with distinct signaling profiles. PLoS One $2012 ; 7$ : 1-14.

10. Congreve M, Langmead CJ, Mason JS, Marshall FH. Progress in structure based drug design for $\mathrm{G}$ protein-coupled receptors. J Med Chem 2011 ; 54 : 4283-311.
11. Bokoch MP, Zou Y, Rasmussen SGF, et al. Ligand-specific regulation of the extracellular surface of a G-protein-coupled receptor. Nature $2010 ; 463$ : 108-14.

12. Ilien B, Glasser N, Clamme JP, et al. Pirenzepine promotes the dimerization of muscarinic $\mathrm{Ml}$ receptors through a three-step binding process. J Biol Chem 2009 ; 284 : 19533-43.

13. Ilien $B$, Franchet $C$, Bernard $P$, et al. Fluorescence resonance energy transfer to probe human $\mathrm{Ml}$ muscarinic receptor structure and drug binding properties. J Neurochem $2003 ; 85: 768-78$.

14. Vollmer JY, Alix P, Chollet A, et al. Subcellular compartmentalization of activation and desensitization of responses mediated by NK2 neurokinin receptors. J Biol Chem $1999 ; 274$ : 37915-22.

15. Maillet $\varepsilon L$, Pellegrini N, Valant C, et al. A novel, conformation-specific allosteric inhibitor of the tachykinin NK2 receptor (NK2R) with functionally selective properties. FASEB J $2007 ; 21: 2124-34$.

16. Palanche $T$, Ilien B, Zoffmann $S$, et al. The neurokinin A receptor activates calcium and cAMP responses through distinct conformational states. J Biol Chem $2001 ; 276: 34853-61$.

17. Sprang S. GEFs: master regulators of G-protein activation. Trends Biochem Sci $2001 ; 26: 266-7$.

18. Strange PG. Agonist binding to G-protein coupled receptors. BrJ Pharmacol $2000 ; 129: 820-1$.

19. Swaminath G, Steenhuis J, Kobilka B, Lee TW. Allosteric modulation of beta2-adrenergic receptor by $\mathrm{Zn}^{2+}$. Mol Pharmacol $2002 ; 61: 65-72$.

20. Paila YD, Chattopadhyay A. The function of $G$-protein coupled receptors and membrane cholesterol: specific or general interaction? Glycoconj J 2009 ; $26: 711-20$.

21. Bockaert J, Fagni L, Dumuis A, Marin P. GPCR interacting proteins (GIP). Pharm Ther $2004 ; 103: 203-21$.

22. Janz JM, Ren $Y$, Looby $R$, et al. Direct interaction between an allosteric agonist pepducin and the chemokine receptor CXCR4. J Am Chem Soc 2011 ; 133 : 15878-81

23. Birdsall NJM, Lazareno S. Allosterism at muscarinic receptors: Ligands and mechanisms. Mini-Rev Med Chem 2005 ; 33 : 523-43.

24. Digby GJ, Shirey JK, Conn PJ. Allosteric activators of muscarinic receptors as novel approaches for treatment of CNS disorders. Mol Biosyst $2010 ; 6$ : 1345-54.

25. Mohr K, Tränkle C, Kostenis $\varepsilon$, et al. Rational design of dualsteric GPCR ligands: quests and promise. BrJ Pharmacol 2010 ; 159 : 997-1008.

26. Tahtaoui C, Parrot I, Klotz P, et al. Fluorescent pirenzepine derivatives as potential bitopic ligands of the human Ml muscarinic receptor.J Med Chem $2004 ; 47: 4300-15$.

27. Valant C, Maillet $\varepsilon$, Bourguignon JJ, et al. Allosteric functional switch of neurokinin A-mediated signaling at the neurokinin NK2 receptor: structural exploration. J Med Chem 2009; 52 : 5999-6011.

28. Urwyler S. Allosteric modulation of Family C G-protein-coupled receptors : From molecular insights to therapeutic perspectives. Pharmacol Rev 2011 ; $63: 59-126$.

29. Goudet C, Gaven F, Kniazeff J, et al. Heptahelical domain of metabotropic glutamate receptor 5 behaves like rhodopsin-like receptors. Proc Natl Acad Sci USA $2004 ; 101$ : 378-83.

30. Schann S, Mayer S, Franchet C, et al. Chemical switch of a metabotropic glutamate receptor 2 silent allosteric modulator into dual metabotropic glutamate receptor 2/3 negative/positive allosteric modulators. J Med Chem $2010 ; 53: 8775-9$.

31. Sebag JA, Pantel J. Ciblage thérapeutique des récepteurs couplés aux protéines $\mathrm{G}$ : la voie allostérique. Med Sci (Paris) $2012 ; 28: 845-51$.

32. Lebon $\mathrm{G}$, Tate $\mathrm{C}$. Les récepteurs couplés aux protéines $\mathrm{G}$ dans la lumière. Med Sci (Paris) $2012 ; 28: 876-82$.

33. Banères JL, Mouillac B. Manipulation des RCPG : expression, purification et stabilisation in vitro. Med Sci (Paris) $2012 ; 28: 837-44$.

34. Jockers R, Gbahou F, Tadagaki K, Kamal M. Oligomérisation des protéines humaines et virales à sept domaines transmembranaires : nouvelle stratégie virale pour manipuler la cellule hôte. Med Sci (Paris) 2012 ; 28 : 864-9.

35. Haga K, Kruse AC, Asada H, et al. Structure of the human M2 muscarinic acetylcholine receptor bound to an antagonist. Nature $2012 ; 482$ : 547-51. 